Yukawa Institute Kyoto

YITP/K-997

December 1992

\title{
Poisson and Porter-Thomas Fluctuations in Off-yrast Rotational Transitions
}

\author{
M.Matsuo $^{a)}$, T.Døssing $^{b)}$, B.Herskind $^{b)}$ and S. Frauendorf $c$ ) \\ a) Yukawa Institute for Theoretical Physics, Kyoto University, Kyoto 606-01, Japan \\ b) Niels Bohr Institute, University of Copenhagen, DK-2100 Copenhagen Ø, Denmark \\ c) Institut für Kern- und Hadronenphysik FZ-Rossendorf, PF 19, O-8054 Dresden, Germany
}

\begin{abstract}
Fluctuations associated with stretched E2 transitions from high spin levels in nuclei around ${ }^{168} \mathrm{Yb}$ are investigated by a cranked shell model extended to include residual two-body interactions. It is found that the gamma-ray energies behave like random variables and the energy spectra show the Poisson fluctuation, in the cranked mean field model without the residual interaction. With two-body residual interaction included, discrete transition pattern with unmixed rotational bands is still valid up to around $600 \mathrm{keV}$ above yrast, in good agreement with experiments. At higher excitation energy, a gradual onset of rotational damping emerges. At $1.8 \mathrm{MeV}$ above yrast, complete damping is observed with GOE type fluctuations for both energy levels and transition strengths (Porter-Thomas fluctuations).
\end{abstract}




\section{Introduction}

Recently, improved detectors and analysis techniques have focused the attention on the off-yrast nuclear structure of deformed nuclei at high spin. For example, it is expected that the rotational band structure typical of the near-yrast regions is smeared out at an intrinsic excitation energy of order of $E_{x} \gtrsim 1 \mathrm{MeV}$ measured from the yrast in rare-earth nuclei ${ }^{1}$ ). There are also arguments that this damping property of the rotational motion is related to onset of chaotic behavior in deformed nuclei ${ }^{2,3,4}$ ).

Information of the nuclear structure of rotating nuclei is usually obtained from identifying the E2 transitions in rotational bands near yrast, while the gamma-rays emitted from levels at higher excitation energy form the so-called "quasi-continuum" spectra in which individual transitions are not distinguished because of the high level density and finite detector resolution. The fluctuation analysis technique, which has been developed recently ${ }^{5}$ ), is useful for a study of the quasi-continuum spectra. From the analysis of fluctuations in the ridge region of 2-D $E_{\gamma}-E_{\gamma}$ spectra, an effective number of the E2-decay paths associated with decay along rotational bands is extracted. The fluctuation analysis technique is based on the assumption that the gamma-ray energies of the rotational E2 transitions behave like random variables, as if they are like rain drops falling at arbitrary positions ${ }^{5}$ ). This is the key assumption in making a connection between the fluctuation of counts in the quasi-continuum spectra and the number of rotational bands passed in the decay.

It should be emphasized that we actually do not know the underlying fluctuation behavior of the E2 transitions. Consider rotational bands in the near-yrast region which do not interact strongly with each other. One may expect that if some dynamical quantum numbers or symmetries persist, they imply regular patterns or correlations in the rotational transition energies. On the other hand, the rotational perturbation at high spin may be able to destroy such regularity by breaking the dynamical symmetries. Hence the validity of assumption of random E2 transition energies, adopted by the fluctuation analysis, may depend on whether any dynamical symmetries in the rotating nuclei survive.

The E2 transitions are expected to show very different fluctuation behavior when the rotational damping sets in. In this case, the E2 transitions from an initial level fragment into many final levels because many rotational bands with different intrinsic configurations are strongly mixed. One may readily expect that the wave functions of 
the strongly mixed levels are very complicated, and that strengths of individual components of the fragmented E2 transitions strongly fluctuate, reflecting the complexity of the mixed wave functions. A description of the strength fluctuation in the strongly mixed levels may be governed by the random matrix model (the Gaussian Orthogonal Ensemble), in which the strength fluctuations show the Porter-Thomas distribution ${ }^{6}$ ). It is not clear, however, whether the Porter-Thomas strength fluctuation is realized in the E2 transition matrix elements in the region in interest, with intrinsic excitation energy of a few $\mathrm{MeV}$.

In this paper we examine theoretically the fluctuations associated with the E2 transitions in rotating nuclei on the basis of a model for the high spin off-yrast levels. In particular we shall investigate whether randomness of the transition energies is realized for the non-interacting rotational bands, and whether the strengths in the damped E2 transitions exhibit Porter-Thomas fluctuations.

\section{Extended cranked shell model}

The model we use is an extension of the cranked shell model, in which we take into account not only the cranked single-particle Hamiltonian but also the two-body residual interactions which cause the rotational damping. As the single-particle fields we adopt the Nilsson potential and the cranking term $-\omega J_{x}$, but neglect the static pairing gaps, since the levels emitting the quasi-continuum gamma-rays are in the high angular momentum region around $30 \hbar$ to $60 \hbar$, where the pairing gap is expected to be negligible.

It is straightforward to introduce the independent-particle configurations using single-particle orbits in the cranked Nilsson field. Many-particle and many-hole $(n \mathrm{p}-n \mathrm{~h})$ excitations should be included to form a suitable basis for the off-yrast levels. The independent-particle configuration is hereafter denoted as $|\mu(\omega)\rangle$. As an ansatz, it is assumed that the $n \mathrm{p}-n \mathrm{~h}$ configurations correspond to the rotational bands which would exists in the off-yrast region if there were no interactions between the rotational bands. A residual two-body interaction $H_{\text {res }}$ is assumed between the rotational bands. Thus the Hamiltonian in this model is expressed:

$$
H=h_{N i l s s o n}-\omega J_{x}+H_{\text {res }}
$$

as a function of the rotational frequency $\omega$ (using the convention $\hbar=1$ ). Details of the 
residual interaction are discussed in sect.4.

The Hamiltonian $H$ is diagonalized within a suitably truncated basis set of $\{|\mu(\omega)\rangle\}$. The diagonalization is separately made among the basis states with the same parity $\pi$ and signature $\alpha$ at fixed $\omega$. The resultant levels are denoted by $i$, their energy by $E_{i}(\omega)$ and their wave functions by $|i(\omega)\rangle$. The routhians of the levels are defined by $e_{i}^{\prime}(\omega)=E_{i}(\omega)-E_{r e f}(\omega)$, where the reference energy $E_{r e f}(\omega)$ is chosen as the lowest energy level in the numerical calculations.

The rotational stretched E2 transitions are calculated as follows. As far as we consider well-deformed nuclei with stable shape, it may be assumed as a good approximation that intra-band transitions dominate if no interaction between the bands exists and the quadrupole moments are the same (constant $Q_{0}$ ) for all the bands. The E2 transition strength and the associated $\gamma$-ray energy can be calculated as

$$
\begin{gathered}
S_{i j}=g Q_{0}^{2}\langle j(\omega-2 / \mathcal{J}) \mid i(\omega)\rangle^{2}, \\
E_{\gamma}=2 \omega+e_{i}^{\prime}(\omega)-e_{j}^{\prime}(\omega-2 / \mathcal{J}) \equiv E_{i j},
\end{gathered}
$$

for a transition between levels $i$ and $j$. Here $|j(\omega-2 / \mathcal{J})\rangle$ is a solution of the extended cranked shell model at the rotational frequency $\omega-2 / \mathcal{J}, \mathcal{J}$ denoting the average moment of inertia. In this way, $i$ and $j$ represent states at angular momentum $I$ and $I-2$, respectively. The factor $g$ in Eq.(2) represents the geometrical factor which arises from the rotational $D$-function. Only the overlap matrix element $\langle j(\omega-2 / \mathcal{J}) \mid i(\omega)\rangle^{2}$ plays a role in the following analysis since we always normalize the E2 strengths.

The above framework is similar to the one employed by S. Aberg ${ }^{3,4}$ ) in many respects. References ${ }^{3,4}$ ), however, introduce an additional procedure to transform the energy of the $n$ p- $n$ h basis $|\mu(\omega)\rangle$ to the lab-frame energy as a function of the angular momentum $I$, and the diagonalization is made at a fixed $I$. We do not follow this procedure here because it is not expected to influence the statistical properties of the results, although individual levels may differ in both descriptions. The procedure in refs. ${ }^{3,4}$ ) may be useful for direct comparison with experiments, especially when describing $E_{\gamma}-E_{\gamma}$ spectra. The procedure described above is a more straightforward theoretical framework and simpler in practical numerical calculations. 
With the residual interactions incorporated, the extended cranked shell model can describe both the rotational bands near the yrast line and the levels far off yrast, where strong bands mixing may occur. Nevertheless, we shall divide our analysis into two parts for convenience of discussion. We first neglect the residual interaction, as in the conventional cranked shell model. The intra-band E2 transitions in the near-yrast regions are then described by this approximation since it is expected that the rotational bands in this region do not mix with each other. We shall discuss the statistical properties for those intra-band rotational transitions in sect. 3. In sect. 4, we discuss the results of the extended cranked shell model where the residual interactions are incorporated, concentrating on the E2 transitions in the far-off-yrast region where the rotational damping is found. In sect. 5 a brief discussion of the transitional region between unmixed and mixed rotational bands is given.

\section{Non-interacting rotational bands}

In the cranked shell model without residual interaction, the rotational bands are simply described as independent-particle configurations assigned to the cranked singleparticle orbits (called $n \mathrm{p}-n \mathrm{~h}$ bands hereafter) and only the intra-band stretched E2 transition is considered. The transition strength is almost constant, irrespective of the configurations of the bands, provided that the deformation is stable. On the other hand, the transition energy varies from band to band because of differences in the alignment. Thus it is appropriate to focus our attention on the fluctuation property of the transition energies.

\subsection{DISTRIBUTION OF $\gamma$-RAY ENERGIES}

The gamma-ray energy of the the stretched intra-band E2 transitions of a $n \mathrm{p}-n \mathrm{~h}$ band is expressed as

$$
\begin{aligned}
E_{\gamma} & =2 \omega_{\mu}(I) \\
& \simeq 2 \omega+\frac{2}{\mathcal{J}}\left(I-J_{\mu}(\omega)\right)
\end{aligned}
$$

in terms of the expectation value $J_{\mu}(\omega)$ of $J_{x}$ for an independent-particle configuration $\mu$ at $\omega$. In expressing the rotational frequency $\omega_{\mu}(I)$ which corresponds to spin value $I$, we make the approximation as $I=J_{\mu}\left(\omega_{\mu}(I)\right) \simeq J_{\mu}(\omega)+\mathcal{J}\left(\omega_{\mu}(I)-\omega\right)$ with $\mathcal{J}$ being the moment of inertia. Eq.(4) can also be derived from Eq.(3). It should be noticed that the stretched E2 transitions occur successively following the spin sequence 
$\cdots I+2 \rightarrow I \rightarrow I-2 \cdots$. In order to investigate the properties of spectra containing all these sequential transitions we displace the $E_{\gamma}$ spectrum by $\pm 4 / \mathcal{J}, \pm 8 / \mathcal{J}$, etc, and fold all the displaced spectra. Here the energy unit $4 / \mathcal{J}$ of the displacement is assumed to be $0.06 \mathrm{MeV}$ which is appropriate for well-deformed nuclei with $A \sim 170$. The folded $E_{\gamma}$ spectra are equivalent to the spectra of $J_{\mu}(\omega)$ modulo 2 , which we denote by $\Delta J_{\mu}$. A typical channel width of the experimental spectra is about one tenth of the $\gamma$-ray energy unit $4 / \mathcal{J}$. Correspondingly, we calculate the distribution of $\Delta J_{\mu}=J_{\mu}(\omega)$ modulo 2 after sorting the $\Delta J_{\mu}$ 's into 10 channels. Figure 1 shows a typical result for ${ }^{168} \mathrm{Yb}$. The Nilsson parameters in ref. ${ }^{7}$ ) and the deformation parameters $\epsilon_{2}=0.255, \epsilon_{4}=0.014$ in ref. $^{8}$ ) are used. Here the spectra in fig. 1 are calculated for the lowest 20 bands (fig.1(a)) with the signature and parity quantum numbers $(+, 0)$ as well as for the next 20 bands, the third 20 bands and so on, altogether 10 bins of bands.

\section{Fig.1}

\subsection{FLUCTUATIONS OF DISCRETE $\gamma$-RAY SPECTRA}

The spectra in fig.1 display a quite random fluctuation. In order to analyze the fluctuation quantitatively, the second moment or the variance of the spectra is calculated in accord with the fluctuation analysis of the experimental data ${ }^{5}$ ). The second moment $\mu_{2}$ here is defined by $\mu_{2}=\left(\sum_{n} m_{n}^{2}\right) / N_{\text {channel }}-\left(\left(\sum_{n} m_{n}\right) / N_{\text {channel }}\right)^{2}$ where $m_{n}$ is the number of $\Delta J_{\mu}$ 's in the $n$-th channel. From the $\mu_{2}$ 's obtained for the 10 bins we calculate the average value at different rotational frequencies $\omega$. The result is plotted in fig.2 as a function of $\omega$. For comparison we show in the same figure the expected limit if the distribution of $\Delta J_{\mu}$ is completely random, i.e. obeys a Poisson distribution. The random limit is calculated by assuming that the distribution of number $m_{n}$ in channel $n$ is binomial. The resulting average value of $\mu_{2}$ is $\left(1-N_{\text {channel }}^{-1}\right) \mu_{1}=1.8$ with the mean number $\mu_{1}=2$, and the statistical sampling allows a deviation within 0.26 . The

standard deviation of $\mu_{2}$ in the limit is $\sigma\left(\mu_{2}\right)=\sqrt{2 / N_{\text {channel }}} \mu_{1}=0.89$. The random limit is indicated in fig. 2 with the horizontal line and the expected deviation with the dashed lines.

Fig.2 
It is clearly seen in fig.2 that the fluctuation in the calculated spectra follows the random limit for rotational frequencies $\omega$ higher than $0.3 \mathrm{MeV}$. It is also seen that there is a systematic deviation from the limit for low rotational frequencies. We find that this deviation arises from the fact that time-reversal doublets in the cranked single-particle orbits are less dissolved for lower $\omega$, because the rotational perturbation is weak. This causes a degeneracy in $\Delta J_{\mu}$, which results in larger fluctuations. It should be noted, however, that the cranked shell model does not take into account effects which influence the spectra at low spin. For example, the intrinsic angular momentum components along the symmetry axis ( $K$ components) is expected to split the degenerate states which have different $K$ values. The effect of the $K$ component on the $\gamma$-ray energy is evaluated according to Eq.(A.5) given in the appendix. We estimate values of $K$ for rotational bands by assigning them to those that are obtained at $\omega=0$. This gives a order-of-magnitude estimate of the $K$ effect. The resulting second moments of the $\gamma$-ray energy spectra are shown in fig.2 as triangles. One can see that the inclusion of the $\mathrm{K}$ effect enhances the tendency toward the random limit. We conclude that the random limit is achieved for the high spin region $\omega \gtrsim 0.3 \mathrm{MeV}$ or $E_{\gamma} \gtrsim 0.6 \mathrm{MeV}$.

It is neccesary to check the above conclusion because our sampling of the rotational bands in the above analysis may be somewhat artificial. We sample the lowest $200 \mathrm{np}$ $n \mathrm{~h}$ bands for each $(\pi, \alpha)$ while the number of the unmixed rotational bands is order of a few tens according to the experimental fluctuation analysis ${ }^{5}$ ). We therefore perform a physically more relevant analysis by sampling only the bands near yrast. From $n \mathrm{p}-n \mathrm{~h}$ bands with all combinations of $(\pi, \alpha)$, the lowest 20 bands are picked up. Associated $\Delta J_{\mu}$ 's are sorted into the channels in the same manner as in fig.1. To obtain a set of the second moments $\mu_{2}$ of spectra, we perform the same calculation for 10 eveneven nuclei around ${ }^{168} \mathrm{Yb}$, namely, ${ }^{162-166} \mathrm{Er},{ }^{164-170} \mathrm{Yb}$, and ${ }^{168-172} \mathrm{Hf}$. From the set of $\mu_{2}$ 's, we calculate the mean value and the standard deviation $\sigma\left(\mu_{2}\right)$ as a function of $\omega$. The result is shown in fig.3, also with the $K$ effect taken into account. From the comparison with the average $\mu_{2}$ and $\sigma\left(\mu_{2}\right)$ of the random Poisson distribution, the following picture emerges: The intra-band $\gamma$-ray energies of the rotational bands in the independent-particle cranked shell model appear to be randomly distributed, at least at high spin. 
Fig.3

\section{Mixed rotational bands}

\subsection{RESIDUAL INTERACTION}

The two-body residual interaction consists of the monopole and quadrupole pairing interaction and the quadrupole-quadrupole interaction, which represent large components of the two-body matrix elements. We also introduce additional components of the two-body matrix elements, which may arise from the other multipolarities. By assuming the additional components are structureless, we give them simply in terms of random numbers following the Gaussian distribution around zero mean value. The strength of the residual interaction is fixed as follows. For the monopole pairing interaction we adopt a standard value $G_{0}=20 / A \mathrm{MeV}$. We fix the quadrupole pairing strength by $G_{2} / G_{0}=35 / 3 R^{4}$ which is derived from the multipole expansion of the delta-force ${ }^{9}$ ). The quadrupole-quadrupole interaction strength is given by the selfconsistent values ${ }^{10}$ ) for both iso-scalar and iso-vector components with the polarization effect $\left(\chi_{n n}=\chi_{p p}=0.7 \chi_{\text {self }}, \chi_{n p}=3.3 \chi_{\text {self }}\right.$ where the $\chi_{\text {self } f}$ is the selfconsistent value $)$. In the numerical calculation, we used the stretched quadrupole operator (instead of the ordinary ones) for practical reasons.

The strength of the random two-body matrix elements is fixed as in ref. ${ }^{1}$ ), namely the spreading width $\Gamma_{\mu}^{\downarrow}=0.039\left(\frac{A}{160}\right)^{-1 / 2} E^{3 / 2} \mathrm{MeV}$ of independent-particle configuration is extracted from the systematics of the single-particle spreading width, and related to the random two-body interaction through the Fermi golden rule $\Gamma_{\mu}^{\downarrow}=2 \pi \rho_{2-\text { body }} v^{2}$. The density $\rho_{2-\text { body }}$ of levels interacting through the two-body interactions is calculated explicitly in the cranking model without the interactions and it is parametrized as $\rho_{2-\text { body }} \simeq 22 E^{3 / 2} \mathrm{MeV}^{-1}$. The root-mean-square value $v$ of the random two-body matrix elements is thus fixed to $v=16 \mathrm{keV}$.

It can be argued that there is an uncertainty in the residual interaction strength. However this uncertainty is not crucial in the following analysis as long as we do not aim at very accurate numbers in the excitation energy. As is discussed in ref. ${ }^{3}$ ), the two-body interaction strength scales proportionally to the spacing between interacting configurations, i.e. $d_{2-\text { body }}=1 / \rho_{2-\text { body }} \propto E^{-3 / 2}$. If the scaling is a good approxima- 
tion, a factor 2 , for example, in the interaction strength results in a scaling of $2^{2 / 3} \sim 1.6$ in the excitation energy. This should, however, only be taken as a guideline since we are investigating properties concerning the full set of states.

\subsection{CHOICE OF BASIS}

In diagonalizing the Hamiltonian matrix the basis of $n \mathrm{p}-n \mathrm{~h}$ configurations is truncated in the following way. First, we construct all the $n \mathrm{p}-n \mathrm{~h}$ configurations that lie below $E_{x}=3.0 \mathrm{MeV}$ above the lowest energy configuration. Next the diagonal energies due to the residual interaction are calculated for these configurations. The basis of the diagonalization consists of the lowest configurations including the diagonal interaction up to a certain number, eg, 600,1000 , or 1400 , for each $(\pi, \alpha)$. The levels at $\omega-2 / \mathcal{J}$ are calculated using the same basis as defined at $\omega$. Figure 4 shows the density of levels calculated thus for the levels with $(-, 1)$ in ${ }^{168} \mathrm{Yb}$ at $\omega=0.5 \mathrm{MeV}$. Results with the three different truncations are plotted to display the convergence. The basis of 1000 states is found to be sufficient for describing the levels up to $2.0 \mathrm{MeV}$ from the yrast, amounting to over 200 levels for each $(\pi, \alpha)$. In the following the calculation with 1000 states is discussed. Most of the figures presented below are for calculation on ${ }^{168} \mathrm{Yb}$ at $\omega=0.5 \mathrm{MeV}$, although a few include the results from the 10 neighboring even-even nuclei ${ }^{162-166} \mathrm{Er},{ }^{164-170} \mathrm{Yb}$, and ${ }^{168-172} \mathrm{Hf}$.

Fig.4

\subsection{TRANSITION STRENGTH FLUCTUATIONS}

When the residual interaction and band mixing are included in the calculations, we see that rotational transitions from each level branch out into many final levels. A clear evidence of this manifestation of rotational damping is shown in fig.5, which depicts four typical examples of the calculated rotational strength distributions plotted as a function of $\gamma$-ray energy. The strengths shown in each quarter correspond to decay from individual levels. The four levels with $(\pi, \alpha)=(-, 1)$ from different regions of excitation energy demonstrate how the rotational decay will change its structure as the excitation energy is increased. 
For the yrast level, the rotational strength concentrates into almost only one transition, indicating the dominance of the rotational band structure. For the 6-th level at $E_{x}=758 \mathrm{keV}$, the strength fragments into several pieces. We may consider this fragmentation as damping of the rotational transitions, although this is rather subtle because of the small number of fragments. The situation becomes clearer for the 24-th level $\left(E_{x}=1.133 \mathrm{MeV}\right)$ and for the 200-th level $\left(E_{x}=1.948 \mathrm{MeV}\right)$ : the rotational strength from these levels splits into a large number of fragments and there appears a smooth profile from which a damping width can be extracted. Thus we see in fig. 5 the onset of rotational damping around several hundred keV.

Next, let us focus our attention on the statistical properties of the rotational transitions, in particular, on those associated with the rotational damping. As seen in fig.5, the height of the peaks or magnitude of the rotational strengths fluctuates significantly around the smooth profile of the strength distributions. This fluctuation of the strength is what would be expected for levels with complex mixing. To clarify the nature of the strength fluctuation, we shall discuss it on a quantitative basis. In particular, we shall discuss whether it exhibits the Porter-Thomas fluctuations or not.

In analyzing the fluctuation in the rotational strength it should be noted that the strength distribution exhibits an overall smooth profile, which may be characterized by the central peak position and the rotational damping width (or FWHM). The strength fluctuation seen in fig.5, therefore, should be measured relative to the smooth profile. It is appropriate to single out the relative fluctuation by normalizing the E2 strengths with respect to the smooth profile. For a transition from $i$ to $j$, the normalized strength is defined by

$$
s_{i j}=\frac{S_{i j}}{\left\langle S_{i j}\right\rangle}
$$

where $\left\langle S_{i j}\right\rangle$ represents the strength in the absence of fluctuations. By definition, the normalized strength should have unit mean. The smoothed strength $\left\langle S_{i j}\right\rangle$ should be a function of the $\gamma$-ray energy $E_{i j}$ and the excitation energy of the levels. One can evaluate it by

$$
\left\langle S_{i j}\right\rangle=\bar{S}\left(E_{i j}\right) / \rho\left(e_{j}^{\prime}\right)
$$


where $\bar{S}(E)$ is the smooth profile of the rotational strength distribution function. The smooth strength function $\bar{S}(E)$ is extracted by taking a Strutinsky-type smoothing of the microscopically calculated strength distribution

$$
S(E)=\sum_{i j} S_{i j} \delta\left(E-E_{i j}\right) / \sum_{i}
$$

where the average over initial levels yields a reliable smoothing. In the Strutinsky smoothing, a Gaussian width of $50 \mathrm{keV}$ is used. The level density $\rho(e)$ is determined by a fitting to the constant temperature formula $\left.{ }^{12}\right) \rho(e)=T^{-1} e^{\left(e-E_{0}\right) / T}$.

\section{Fig.6}

In order to see the excitation energy dependence, the initial levels with the same $(\pi, \alpha)$ are grouped into bins, each of which contains 50 levels. The excitation energies of these bins are approximately $0.0-1.4 \mathrm{MeV}$ for $1-50$-th levels, $1.4-1.7 \mathrm{MeV}$ for 51 - 100-th, $1.8 \mathrm{MeV}$ for 101 - 150-th, and $1.9 \mathrm{MeV}$ for 151 - 200-th. The rotational strength distribution calculated for these bins are shown in fig.6 as well as the smoothed strength function $\bar{S}(E)$ plotted with the solid curves. We sample the normalized strengths of the transitions whose $\gamma$-ray energy $E_{i j}$ lies within the interval of the full width at the one eighth of maximum of $\bar{S}(E)$. For the lowest bin, for example, those transitions picked up satisfy $881 \mathrm{keV}<E_{i j}<1170 \mathrm{keV}$.

\section{Fig.7}

After the sampling we calculate distribution of the normalized strength $s_{i j}$. The result is shown in fig. 7 , together with a Porter-Thomas distribution

$$
P(s) d s=(2 \pi s)^{-1 / 2} e^{-s / 2} d s
$$

for comparison. It is clear from fig.7 that that the distribution of the normalized strengths approaches that of Porter-Thomas for increasing excitation energy and almost reaches it in the region $E_{x} \gtrsim 1.8 \mathrm{MeV}$. Deviation from the Porter-Thomas is seen in the lowest bin which corresponds to $E_{x}=0.0-1.4 \mathrm{MeV}$ (also slightly in the next bin). 
In the above analysis it has implicitly been assumed that the property of the strength fluctuation does not depend on the $\gamma$-ray energy. Let us show this for the highest bin which clearly displays Porter-Thomas fluctuations. For this purpose we subdivide the samples of the normalized strength into two groups according to $\gamma$-ray energy. One subgroup is for the transitions whose $\gamma$-ray energies are close to the central peak of the rotational strength function. In practice we choose the transition energies within the Full-Width-Half-Maximum of the smoothed strength function. The other is for transition energies which lie in the tail portion of the strength function (between the half and one eighth of the maximum value). Figure 8 shows the result of this analysis for transitions in the fourth bin $\left(151-200\right.$-th levels at $E_{x} \sim 1.9 \mathrm{MeV}$ in $\left.{ }^{168} \mathrm{Yb}\right)$ of figs. 6 and 7. Regardless of whether the transitions are from the central or from the tail portions, the strength fluctuation is found to obey the Porter-Thomas distribution.

It should be noticed here that the Porter-Thomas distribution manifest itself only for the relative strengths which are normalized with respect to the smooth profile of the rotational strength function. This explains why the Porter-Thomas distribution emerges here while it does not in the analysis made in ref. ${ }^{4}$ ).

\subsection{BRANCHING NUMBER}

Let us analyze the strength fluctuation again, but this time by looking into it in terms of a quantity which is closely related to the experimental observables. In the experimen-

tal fluctuation analysis ${ }^{5}$ ) the number of the rotational decay paths $n_{\text {path }}^{(2)}$ is extracted from the second moment of the spectral fluctuation. Assuming one-dimensional spectra (or observing one-step transitions), the number of paths is given by

$$
\begin{gathered}
\frac{1}{n_{\text {path }}^{(2)}}=\sum_{i} \frac{f_{i}^{2}}{n_{\text {branch }}(i)}, \\
n_{\text {branch }}(i)=\left(\sum_{j} w_{i j}^{2}\right)^{-1},
\end{gathered}
$$

where $f_{i}$ represents the probability for flow passing through the level $i$, and $w_{i j}$ the probability for the rotational transition to pass from level $i$ to level $j$ (with normalization 
$\left.\sum_{j} w_{i j}=1\right)$. The quantity $n_{\text {branch }}(i)$ is a measure of the number of transitions which branch out of level $i$ (or simply the branching number). With the microscopic model the branching numbers are determined by the transition probability as given by

$$
w_{i j}=S_{i j} / \sum_{j} S_{i j}
$$

where the energy dependent factor $\propto E_{\gamma}^{5}$ is neglected. On the other hand, the average branching number expected for a group of levels whose E2 strength displays PorterThomas fluctuations can be estimated analytically by using a suitable profile of the rotational strength function. In fact, the Gaussian form with FWHM of the rotational damping width $\Gamma_{\text {rot }}$ is adopted. The average branching number in the Porter-Thomas limit is then given by

$$
\begin{aligned}
\overline{n_{\text {branch }}(i)_{, \mathrm{PT}}} & =\frac{1}{6} \sqrt{\frac{2 \pi}{\ln 2}} \Gamma_{\text {rot }} \rho\left(E_{i}\right) \\
& \approx 0.5 \Gamma_{\text {rot }} \rho\left(E_{i}\right) .
\end{aligned}
$$

$$
\text { Fig.9 }
$$

Figure 9 compares the Porter-Thomas limit Eq.(12) with the average branching number calculated microscopically by means of Eq.(10). In Eq.(12) we adopt $\Gamma_{\text {rot }}=200$ $\mathrm{keV}$. This value agrees well with the FWHM's extracted from the smoothed strength function shown in fig.6. Actually they are approximately $170 \mathrm{keV}$ and the agreement is fulfilled within an accuracy of $15 \%$. The validity of Eq. (12) supports again that the Porter-Thomas fluctuation is realized in the damped rotational transitions.

\subsection{ENERGY LEVEL FLUCTUATIONS}

It may be interesting to look into the fluctuation in the energy levels because it is expected to show the properties of the GOE random matrix theory if the PorterThomas strength fluctuation is a consequence of the complex mixing of the rotational bands. Here we test the $\Delta_{3}$ statistics ${ }^{6}$ ) as a measure of the energy level fluctuations. The calculated spectra are unfolded for each $(\pi, \alpha)$ by use of a fitted level density, parametrized according to the constant temperature formula. An example of the fitting is shown in the inset of fig. 10. The mean value of $\Delta_{3}$ is calculated as a function of 
the interval length $L$ (the unit is the average spacing). As in the preceding section, we subdivided the levels into bins of 50 levels.

Fig.10

Figure 10 shows that the $\Delta_{3}$ statistics approaches the GOE limit with increasing excitation energy and that the limit is reached for the levels above \#150 at $E_{x} \sim 1.8$ $\mathrm{MeV}$. It is remarkable, compared to the results shown in fig.7, that the tendency toward the GOE limit is completely consistent with that of the strength fluctuation. Thus it can be concluded, from both the strength fluctuations and the energy level fluctuations, that the mixing of the rotational bands is as complex and random in the energy region $E_{x} \gtrsim 1.8 \mathrm{MeV}$ as described by the GOE random matrix theory. Concerning the energy level fluctuations, the results are consistent with the work by S. Åberg ${ }^{3,4}$ ).

We would like to emphasize the important implication of the above result for the rotational transitions in the damping region. A spectrum which shows GOE fluctuations is known to be "rigid" with very small fluctuations. As a consequence, the fluctuations

in transition energy play a relatively unimportant role in the damping region, while the strength fluctuation becomes the dominant origin of the spectral fluctuations ${ }^{13}$ ).

\section{Onset of damping}

As discussed in connection with fig.5, the model predicts both the damped transitions and the discrete rotational transitions which characterize unmixed rotational bands near yrast. Thus it allows us to investigate the onset of the rotational damping and also to analyze fluctuation properties of the discrete rotational transitions, which was discussed in the preceding section without including the residual interaction.

Fig.11

\subsection{UNMIXED ROTATIONAL BANDS}

Figure 11 illustrates the behavior of rotational transitions in the energy region where the damping is about to set in. The branching numbers calculated for individual levels are also shown in the figure. A quantitative criterion for defining levels associated with 
unmixed rotational bands may be given by a condition that the branching number of a level is less than 2. Accordingly the onset of the damping will be marked by $n_{\text {branch }}>2$. If this criterion is assumed we count 22 unmixed rotational bands in ${ }^{168} \mathrm{Yb}$, and 30.9 bands on average for the 10 rare-earth nuclei around spin $42 \hbar$. Considering that the pure rotational bands carry essentially $100 \%$ strength of the intra-band transition, one may also define unmixed rotational-band levels as those whose strongest transition has a large fraction of the total strength, e.g. $1 / \sqrt{2}=71 \%$. This gives us gives us 19 rotational bands for ${ }^{168} \mathrm{Yb}$ and 28.3 if averaged over the 10 neighboring nuclei. The two criteria give almost the same number. The above number of the unmixed rotationalband levels is consistent with the experimental fluctuation analysis ${ }^{5}$ ), which reports approximately 30 bands in ${ }^{168} \mathrm{Yb}$.

Since the residual interaction effect was not included in the preceding analysis using the independent-particle cranked shell model in sect. 3, we re-examine the fluctuation property of the transition energies associated with the discrete rotational transitions defined by the above criterion. For this purpose, we select the lowest 20 levels which satisfy $n_{\text {branch }}<2$ and use their strongest transitions. The second moment $\mu_{2}$ which represents the fluctuation of the selected transition energies is calculated for each nucleus at $\omega=0.5 \mathrm{MeV}$ in the same way as in sect. 3. The average value of $\mu_{2}$ 's over the 10 nuclei is found to be 2.16 , and their standard deviation is 0.80 . These numbers compare well with the random Poisson limits, 1.80 and 0.89, respectively. Thus the residual interaction does not modify the conclusion of sect.3, concerning the randomness of transition energies.

\subsection{TRANSITION STRENGTH FLUCTUATIONS}

The energy for the onset of the rotational damping may be defined although the damping sets in rather gradually with increasing excitation energy as seen in fig. 11. From fig. 9, which shows average excitation energy dependence of the branching number,

and with the criterion $n_{\text {branch }}>2$, the onset energy for ${ }^{168} \mathrm{Yb}$ can be estimated to be approximately $E_{x} \approx 600 \mathrm{keV}$ above yrast.

Above the onset energy, the transitions from each level begin to branch into many small pieces and strong fluctuations in the strengths of the fragmented transitions set in. Although in fig.7 almost pure Porter-Thomas fluctuations are found in the energy region $E_{x} \gtrsim 1.8 \mathrm{MeV}$, a clear deviation from Porter-Thomas is found for lower exci- 
tation energies. To describe the energy dependence of the strength fluctuation more quantitatively, the lowest 50 levels are subdivided into smaller bins of 10 levels and the distribution of the normalized strengths is calculated for each bin, producing the result shown in fig.12. In this figure, results for 10 nuclei are included to obtain good enough statistics. It is seen in fig. 12 that deviations from Porter-Thomas fluctuations are more significant at lower excitation energies. Strong deviations are found not only in the lowest bin $\left(E_{x} \simeq 0.0-0.9 \mathrm{MeV}\right)$ which contains the unmixed rotational-band levels but also in the other bins where the rotational damping becomes dominating. This indicates the presence of a transient region between $0.6 \mathrm{MeV}$ and $1.8 \mathrm{MeV}$ where the rotational transitions are already fragmented significantly, while the strength fluctuations have not yet reached the Porter-Thomas limit.

Fig.12

\subsection{LEVEL SPACING STATISTICS FOR UNMIXED BANDS}

From the viewpoint of discrete spectroscopy ${ }^{14}$ ), fluctuation properties in the energy levels near the yrast line are of interest, although the spin region in the present analysis is higher than current discrete spectroscopies can reach. Figure 13 displays the nearest neighbor level spacing distribution for the rotational-band levels near yrast. As a reference, a result for cranked shell model without the residual interaction is also shown. In this figure only the lowest energy levels satisfying $n_{\text {branch }}<2$ (the lowest 5 levels for the independent-particle model) are sampled for each $(\pi, \alpha)$ from the 10 nuclei. This gives 121 spacings in the case of two-body interaction included, and 160 spacings for the independent-particle model. The energy dependence of the fitted level density is taken into account in normalizing the spacings (cf. sect.4.5 and fig.10). The result for the independent particle model resembles the Poisson distribution. With the residual interaction included, on the other hand, there are less small spacings than expected from the Poisson distribution. The level spacing distribution appears to lie in between the Poisson and the GOE limits.

Fig.13 


\section{Conclusion}

The E2 transitions in the off-yrast region of well-deformed rare-earth nuclei are investigated on the basis of an extended cranked shell model which describes the $n \mathrm{p}$ $n \mathrm{~h}$ bands as well as the mixing among them. Using the cranked shell model without the residual interaction, the fluctuation in the gamma-ray energies of the rotational transitions associated with the near-yrast non-interacting rotational bands are discussed. It is found that the gamma-ray energy spectra at high spin show Poisson fluctuations as if the gamma-ray energies were random variables. The model with the residual interaction is found to give rise to the damping of the rotational transition above an excitation energy $\left(E_{x} \simeq 600 \mathrm{keV}\right)$. It is seen that the magnitude of the $\mathrm{E} 2$ strength fluctuates for the fragmented components of the damped rotational transitions. Further up in excitation energy $\left(E_{x} \gtrsim 1.8 \mathrm{MeV}\right)$, the strength fluctuations are found to obey the Porter-Thomas distribution. In the same energy region, it is also found that the energy level fluctuations distribute according to the GOE random matrix model implying that the mixing of rotational bands is as complex as in the random matrix theory. Using the properties of the random matrix theory as a criterion for the chaotic behavior of quantum systems ${ }^{15}$ ), this region appears to be chaotic. The energy region just above the onset of damping appears as a transient region where the strength fluctuation show significant deviations from the Porter-Thomas distribution.

In the model we adopted, the boundary energy for the onset of rotational damping is about $600 \mathrm{keV}$, and the boundary for sharing full Porter-Thomas strength fluctuation is around $1.8 \mathrm{MeV}$. These numbers, however, should be considered with reservation since they may depend on the residual interaction as well as its strength. It is therefore important to extract these energies in more detailed experiments.

On the theoretical side, it seems interesting to study how the situation changes when more realistic residual interactions are used. Another interesting aspect of band mixing concerns the correlations in the wave functions, which extend over several steps in angular momentum. These correlations are relevant for a more precise comparison to two and higher fold experimental spectra. Such investigations are in progress. 


\section{References}

1) B.Lauritzen, T.Døssing and R.A.Broglia, Nucl. Phys. A457(1986) 61.

2) T.Guhr and H.A. Weidenmüller, Ann. Phys. 193 (1989) 489.

3) S.Åberg, Phys. Rev. Lett. 64(1990) 3119.

4) S.Åberg, Prog. Part. Nucl. Phys. vol.28 (Pergamon 1992) p.11.

5) B.Herskind, A.Bracco, R.A.Broglia, T.Døssing, A.Ikeda, S.Leoni, J.Lisle, M.Matsuo, and E.Vigezzi, Phys. Rev. Lett. 68(1992) 3008.

B.Herskind, T.Døssing, S.Leoni, M.Matsuo, and E.Vigezzi, Prog. Part. Nucl. Phys. Vol.28 (Pergamon 1992) p.235.

6) C.E. Porter and R.G. Thomas, Phys. Rev. 104(1956)483.

T.A.Brody, J.Flores, J.B.French, P.A.Mello, A.Pandy and S.S.M.Wong, Rev. Mod. Phys. 53(1981) 385.

7) T.Bengtsson and I.Ragnarsson, Nucl. Phys. A436(1985) 14.

8) R.Bengtsson, S.Frauendorf and F.-R.May, Atomic Data and Nuclear Data Tables, 35(1986) 15.

9) I.Hamamoto, Nucl. Phys. A232(1974) 445.

10) A.Bohr and B.R.Mottelson, Nuclear structure vol.2 (Benjamin 1975).

11) S.Åberg, Nucl. Phys. 477 (1988) 18.

12) A.Gilbert and A.G.W.Cameron, Can. J. Phys. 43 (1965) 1446.

13) P.G.Hansen, B.Jonson and A.Richter, Nucl. Phys. A518 (1990) 13.

14) J.D. Garrett, J.R. German, L. Courtney, and J.M. Espino, Proc. Symp. on Future Directions in Nuclear Physics with $4 \pi$ Gamma Detection Systems of the New Generation, Strasbourg, 1991, ed. J. Dudek and B. Haas (American Institute of Physics, 1992) p.345.

15) O. Bohigas, M.J. Giannoni and C. Schmit, Phys. Rev. Lett. 52(1984) 1.

O. Bohigas and H.A. Weidenmüller, Ann. Rev. Nucl. Part. Sci. 38 (1988) 421.

\section{Acknowledgments}

One of the authors, MM, acknowledges the Nishina Memorial Foundation for supporting his stay at the Niels Bohr Institute, where parts of this work has been carried out. Illuminating discussions with S. Åberg are gratefully acknowledged. 


\section{Appendix}

With the presence of a $K$ component of the angular momentum, the rotational frequency along the rotational axis can be defined by

$$
\begin{gathered}
\omega(I)=\frac{E(I+1)-E(I-1)}{I_{x}(I+1)-I_{x}(I-1)}, \\
I_{x}(I)=\sqrt{I^{2}-K^{2}} .
\end{gathered}
$$

For a $n \mathrm{p}-n \mathrm{~h}$ band in the extended cranked shell model, in which the angular momentum $I_{x}$ along the rotational axis is calculated as a function of $\omega$ as $I_{x}=J_{\mu}(\omega)$, Eq.(A.2) is used to determine $\omega$ which corresponds to the angular momentum $I$.

$$
\sqrt{I^{2}-K_{\mu}^{2}}=J_{\mu}\left(\omega_{\mu}(I)\right) \simeq J_{\mu}(\omega)+\mathcal{J}\left(\omega_{\mu}(\mathcal{I})-\omega\right)
$$

where $K_{\mu}$ is the $K$ value for the band $\mu$ and $\omega$ is a reference frequency. Eq.(A.1) reduces to an equation which gives the associated $\gamma$-ray energy,

$$
E_{\gamma} \simeq 2 \omega_{\mu}(I) \frac{\partial I_{x}}{\partial I}=2 \omega_{\mu}(I) \frac{I}{\sqrt{I^{2}-K_{\mu}^{2}}}
$$

Combining (A.3) and (A.4), we get

$$
\begin{aligned}
E_{\gamma} & =2 \omega \frac{I}{\sqrt{I^{2}-K_{\mu}^{2}}}+\frac{2}{\mathcal{J}}\left(I-J_{\mu}(\omega) \frac{I}{\sqrt{I^{2}-K_{\mu}^{2}}}\right) \\
& \simeq 2 \omega \frac{\sqrt{J_{\mu}(\omega)^{2}+K_{\mu}^{2}}}{J_{\mu}(\omega)}+\frac{2}{\mathcal{J}}\left(I-\sqrt{J_{\mu}(\omega)^{2}+K_{\mu}^{2}}\right) .
\end{aligned}
$$

In deriving the final expression, we neglect the $I$-dependence in the geometrical factor $I / \sqrt{I^{2}-K_{\mu}^{2}}$ by replacing it with $\sqrt{J_{\mu}(\omega)^{2}+K_{\mu}^{2}} / J_{\mu}(\omega)$. Eq.(A.5) can be used in place of Eq.(4). 


\section{Figure captions}

Fig.1 The spectra of $\Delta J_{\mu}=J_{\mu}$ modulo 2 of the basis $n$ p- $n$ h configurations for $(\pi, \alpha)=$ $(+, 0)$ bands in ${ }^{168} \mathrm{Yb}$ at $\omega=0.5 \mathrm{MeV}$. The first bin contains the first 20 bands in order of the routhian energies, the second bin is for the next 20 bands, and so on.

Fig.2 The averaged second moment $\mu_{2}$ of fluctuation defined for the $\Delta J_{\mu}$ spectra of the $n$ p- $n$ h basis bands with $(+, 0)$ in ${ }^{168} \mathrm{Yb}$ as a function of the rotational frequency $\omega$, plotted with closed circles. The value at $\omega=0.5 \mathrm{MeV}$ corresponds to the spectra shown in Fig.1. The triangles are calculated for the spectra in which the effect of the $K$ components on the $\gamma$-ray energy is taken into account. The random (Poisson) limit is indicated by the horizontal line. The dashed lines represents the uncertainty expected for the average $\mu_{2}$ in the random limit.

Fig.3 The second moment $\mu_{2}$ of fluctuation in $\Delta J_{\mu}$ spectra for the near-yrast $n \mathrm{p}-n \mathrm{~h}$ bands. The lowest twenty bands are sampled from each nucleus. The average value of the $\mu_{2}$ 's calculated for the ten nuclei (see text) is plotted with closed circles. The bars represent the standard deviation of $\mu_{2}$ 's. The random limit for the average $\mu_{2}$ is indicated by the solid line and the thick bar indicate the limit standard deviation.

Fig.4 The density of levels calculated in the extended cranked shell model for the levels with $(-, 1)$ in ${ }^{168} \mathrm{Yb}$ at $\omega=0.5 \mathrm{MeV}$. Results with three different truncations (number of basis states 600, 1000, and 1400) are plotted. The Fermi-gas formula 11) with the level density parameter $a=15.3 \mathrm{MeV}^{-1}$ is also plotted with a dashed curve for comparison.

Fig.5 The calculated rotational E2 strength distribution as a function of the $\gamma$-ray energy $E_{\gamma}$. The four figures correspond to the transitions decaying from the first, 6-th, 24-th, and 200-th levels with $(-, 1)$ in ${ }^{168} \mathrm{Yb}$ at $\omega=0.5 \mathrm{MeV}$, respectively. The magnitude of the strength is given as the fraction relative to the total strength.

Fig.6 The rotational strength distributions are shown for transitions from 50 levels averaged in each bin. The left-top quarter is for the first bin (from the first to the 50 -th level) with $(-, 1)$. The other three quarters are for the levels from 51 -th to 100-th, 101-th to 150-th and 151-th to 200-th. The excitation energy from the yrast line is indicated for each bin. The smoothed distribution function is drawn with a solid curve. The transitions shown are for $(-, 1)$ in ${ }^{168} \mathrm{Yb}$ at $\omega=0.5 \mathrm{MeV}$. 
Fig.7 The distribution of the normalized rotational strengths $s_{i j}$ for ${ }^{168} \mathrm{Yb}$ at $\omega=0.5$ $\mathrm{MeV}$. For convenience we draw the distribution as a histogram against the squareroot $\sqrt{s_{i j}}$ of the normalized strength. In this representation the Porter-Thomas distribution, shown as dashed curves, exhibits Gaussian shape.

Fig.8 The distribution of the normalized rotational strengths. In this figure, the transitions employed in the right-bottom quarter of Fig.7 is divided into two groups according to whether their transition $\gamma$-energies are in the central part of the rotational strength function or in the tail part.

Fig.9 The branching number is shown as a function of the excitation energy from the yrast. The histogram is the result of the microscopic calculation while the dashed curve represents the Porter-Thomas limit given by Eq.(12) in which the rotational damping width $\Gamma_{\text {rot }}$ of $200 \mathrm{keV}$ is adopted. The values are calculated for ${ }^{168} \mathrm{Yb}$ at $\omega=0.5 \mathrm{MeV}$.

Fig.10 The $\Delta_{3}$ statistics of the energy level fluctuation associated with the routhian eigenvalues $e_{\alpha}^{\prime}(\omega)$ in ${ }^{168} \mathrm{Yb}$ at $\omega=0.5 \mathrm{MeV}$. The eigenvalues are grouped into four bins in the same way as in Figs.6 and 7. The long-dashed and the dashed curves represent the limits of the Poisson distribution and the GOE random matrix model, respectively. The fitted level density for $(-, 1)$ is shown in the inset with the adjusted parameters of the constant temperature formula.

Fig.11 The E2 strength distributions as a function of the $\gamma$-ray energy for the lowest 9 levels with $(\pi, \alpha)=(+, 1)$ in ${ }^{168} \mathrm{Yb}$ at $\omega=0.5 \mathrm{MeV}$. The branching number as well as the energy above yrast are also shown for each level.

Fig.12 The distribution of the normalized rotational strengths $s_{i j}$, where the lowest 50 levels for each $(\pi, \alpha)$ are grouped into smaller bins of 10 levels. The approximate energy regions corresponding to the bins are also shown. In this figure the transitions in the 10 nuclei around ${ }^{168} \mathrm{Yb}$ are sampled.

Fig.13 Distribution of the normalized nearest neighbor level spacings $S$ for the unmixed rotational-band levels, defined by $n_{\text {branch }}<2$, near the yrast at $\omega=0.5 \mathrm{MeV}$ (the upper half). The result for the independent-particle cranked shell model without the residual interaction at the same $\omega$ is shown in the lower half. The spacings are sampled from the ten nuclei around ${ }^{168} \mathrm{Yb}$ and are normalized to give $\langle S\rangle=1$. 